\title{
RELAÇÃO ENTRE RESPONSABILIDADE SOCIAL INTERNA E SAÚDE E SEGURANÇA OCUPACIONAL: UMA REVISÃO DA LITERATURA ESTRUTURADA.
}

\section{RELATIONSHIP BETWEEN INTERNAL SOCIAL RESPONSIBILITY AND OCCUPATIONAL HEALTH AND SAFETY: A REVIEW OF STRUCTURED LITERATURE}

\author{
Sávio Luís Oliveira da Silva ; Osvaldo Luiz Gonçalves Quelhas²; \\ ${ }^{1}$ Universidade Federal Fluminense (UFF) - Niterói - RJ - Brasil \\ contato@savioluis.com \\ ${ }^{2}$ Universidade Federal Fluminense (UFF) - Niterói - RJ - Brasil \\ quelhas@latec.uff.br
}

\begin{abstract}
Resumo
Organizações socialmente responsáveis devem prezar, primeiramente, pela saúde e bem estar do seu público interno (empregados), antes mesmo de realizar ações que beneficiem o público externo. Neste sentido, a Ergonomia pode ser de fundamental importância no constructo de condições favoráveis ao trabalho e de melhorias na saúde e segurança do trabalhador. A interseção entre Responsabilidade Social Organizacional (RSO) e Saúde e Segurança Ocupacional (SSO) propõe um tema importante a ser abordado em pesquisas atuais e futuras, na medida em que os aspectos referentes às condições de trabalho são cada vez mais latentes. A presente pesquisa pode ser caracterizada como uma desk research, ou seja, baseou-se em dados secundários e teve como objetivo identificar correlações entre os conceitos de RSO e SSO. No total foram analisados 49 artigos publicados entre os anos de 1981 e 2014 nos periódicos do Portal Capes, nas bases Scopus $e$ Web of Science. Observou-se como principais resultados que a dimensão interna da RSO representa $44 \%$ dos artigos publicados e destes 53\% enfocam a Ergonomia e a (SSO). Algumas lacunas de pesquisa surgiram após este estudo, fato que propõe pesquisas futuras sobre o assunto.
\end{abstract}

Palavras-chave: responsabilidade social organizacional interna; gestão da saúde e segurança ocupacional; sustentabilidade.

\section{Introdução}

Sofrer lesões no trabalho ou ser acometido por doenças associadas às funções laborais pode fazer com que o trabalhador associe estes males à um ambiente de trabalho pouco seguro, contribuindo para a insatisfação, estresse e maior turnover (MCCAUGHEY et al, 2013). Além disso, os indicadores de fadiga e capacidade para o trabalho derivam de vários fatores relacionados às condições de trabalho e à sua organização (METZNER; FISHER, 2010).

No entanto, alguns empregadores não estão realmente preocupados com a proteção de seus 
empregados, e pior ainda, alguns empregadores nem sequer percebem que eles têm a responsabilidade moral e, muitas vezes legal, de proteger seus empregados (MONTERO et al, 2009). Em muitos casos, os gestores terceirizam a responsabilidade pela saúde e segurança do trabalhador para um consultor ou departamento específico para cuidar do assunto (DUL; NEUMANN, 2009).

Dada à relevância do problema, nota-se uma relação entre Responsabilidade Social Organizacional (RSO) e Saúde e Segurança Ocupacional (SSO) um campo interessante a ser abordado em pesquisas atuais e futuras, na medida em que os aspectos referentes às condições de trabalho são imprescindíveis tanto do ponto de vista de reputação da organização quanto de maximização da produtividade.

Desta forma, o presente artigo dispõe do seguinte objetivo geral de pesquisa: "Identificar correlações entre os conceitos de RSO e SSO”.

Para tanto, fazem-se necessários os seguintes objetivos específicos:

- Delimitar adequadamente o escopo da coleta de dados, visando coletar uma quantidade significativa de artigos no âmbito da RSO e SSO;

- Categorizar os referidos artigos em subcategorias correlatas com o tema principal de pesquisa;

- Identificar as interseções entre as subcategorias estudadas, com atenção especial à RSO Interna e SSO.

O presente trabalho foi estruturado em cinco seções. Esta Introdução, de caráter preliminar, apresenta o objetivo da pesquisa. A seção 2 insere o leitor no contexto investigativo proposto no trabalho. Na seção 3, são apresentados os procedimentos metodológicos utilizados. A seção 4 apresenta a ilustração do processo de seleção do constructo teórico de pesquisa e a seção 5 apresenta as conclusões, tecendo reflexões e fazendo recomendações para futuras pesquisas, com base nas limitações do estudo atual.

\section{Contextualização de tema}

É crescente o interesse das organizações e dos centros de pesquisa pelo tema Responsabilidade Social Organizacional (RSO) ao longo dos últimos dez anos, com a literatura abordando o tema em franca ascensão (JONES et al, 2005; FARIA; SAUERBRONN, 2008).

Apesar deste evidente interesse, ainda não existe consenso sobre o que de fato pode ser incluído de forma consistente neste conceito (KOSKELA, 2014). Dentre as várias definições existentes, destaca-se a delineada pela Norma ISO 26000 (ABNT NBR ISO 26000:2010), a qual estabelece as diretrizes sobre responsabilidade social através de um escopo amplo que incorpora a 
dimensão social, ambiental e econômica do desenvolvimento sustentável (COSTA, 2011). Tal Norma fornece a seguinte definição de RSO:

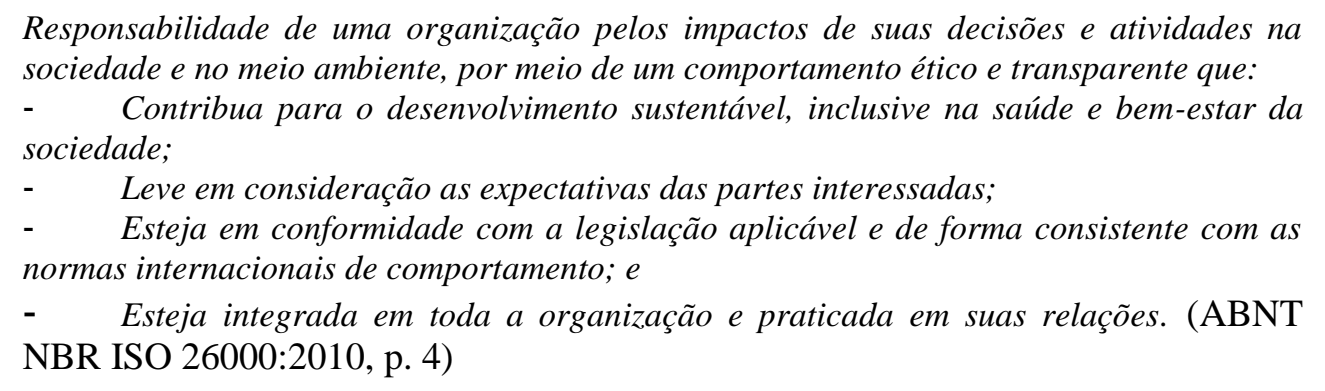

A Responsabilidade Social relaciona-se com os impactos provocados pela empresa na vida das pessoas a partir de duas perspectivas: a interna e a externa (KOSKELA, 2014). As práticas de RSO Interna, segundo Al-bdour et al (2010), estão diretamente relacionadas com os aspectos físicos e psicológicos do ambiente de trabalho. Preocupações com a promoção da saúde e segurança do trabalhador, com a igualdade de oportunidades, com o treinamento e com a relação trabalho-família fazem parte deste escopo. Quanto à RSO Externa, Al-bdour et al (2010) citam o envolvimento da organização com a comunidade local, com os fornecedores, clientes, autoridades públicas e o meio ambiente, por exemplo.

A concepção da RSO antes de ser considerada uma ferramenta gerencial que focalize o ambiente externo, deve prezar pelo ambiente interno como maneira de ampliar e legitimar as práticas socialmente responsáveis (CRUZ, 2012). O autor realça ainda a importância de a organização servir de exemplo, ou seja, iniciar em seu próprio interior as ações de responsabilidade social, para posteriormente avançar perante a comunidade e a sociedade.

Apesar do interesse contínuo em RSO e sua relação com diferentes grupos de interesse, os seus efeitos sobre o trabalho dos trabalhadores têm recebido atenção limitada (MUELLER et al, 2012). Corroborando com tal argumento, Cruz (2012) apresenta o resultado da auto avaliação acerca de práticas organizacionais de RSO, produto de pesquisa que organizações filiadas ao Instituto Ethos organizam anualmente. Nessas pesquisas anuais as organizações reconhecem que suas atuações perante o público interno são uma das mais incipientes, em comparação com as atuações referentes aos demais stakeholders.

À empresa não é permitido relacionar-se com o trabalhador como se fosse uma máquina, muito menos como um indivíduo que deixa seus problemas do lado de fora das fronteiras da organização (FUKUNAGA, 2009). Sendo (MONTERO et al, 2009) afirmam que a preocupação com o bemestar dos funcionários deve constituir um dos principais aspectos da RSO de qualquer empresa, definindo a SSO como uma disciplina ampla que abrange: a promoção e manutenção do mais alto grau de saúde física, mental e bem-estar social dos trabalhadores; a prevenção de problemas de 
saúde causados pelas condições de trabalho; a proteção dos trabalhadores contra os riscos adversos à saúde e a adaptação do ambiente de trabalho de acordo com a capacidade fisiológica e psicológica dos trabalhadores.

A importância da segurança no trabalho para evitar danos às pessoas e à economia é amplamente reconhecida (HADJIMANOLIS; BOUSTRAS, 2013). Segundo o autor, altos níveis de segurança contribuem para uma maior motivação dos funcionários, melhorias na qualidade do produto, na produtividade e no desempenho geral das empresas. Desta forma, tendo por base a importância na promoção de SSO, torna-se extremamente relevante um estudo detalhado da relação trabalho/trabalhador, visando logicamente proporcionar àqueles que executam as tarefas laborais menores riscos e maior qualidade de vida no trabalho. Isso eleva também a importância do estudo da Ergonomia, já que a essência do seu significado a coloca como uma importante área de estudos a ser utilizada pelos gestores no intuito de proporcionar ao seu empregado condições adequadas para desempenhar suas funções.

O aperfeiçoamento das intervenções ergonômicas nos locais de trabalho desempenham um importante papel nas práticas de saúde e segurança ocupacional (KOGI, 2012). Segundo o autor, as ações orientadas neste sentido podem reduzir os riscos relacionados ao trabalho nas mais variadas situações. Os acidentes de trabalho, por exemplo, constituem o principal agravo à saúde dos trabalhadores, com elevados custos sociais e econômicos e que podem chegar a 10\% do Produto Interno Bruto (PIB) (VILELA et al, 2012). Para Ricart et al, 2012, o conceito de promoção de saúde está relacionado com ações que visam intervir na prevenção de doenças no local de trabalho, tanto no aspecto individual quanto no coletivo. Esta intervenção deve incluir atitudes participativas e multidimensionais no intuito de promover tais mudanças.

\section{3- Método de pesquisa}

A presente pesquisa pode ser caracterizada como uma desk research, ou seja, baseou-se em dados secundários e visou coletar e analisar artigos cuja temática geral tratava RSO e a SSO . No total foram analisados 49 artigos publicados entre os anos de 1981 e 2014 nos periódicos listados no Quadro 3.

A coleta dos artigos foi direcionada por um conjunto de palavras-chave que remetem a questões relacionadas à RSO e SSO. Para uma melhor adequação aos estudos, e considerando que nos portais de periódicos escolhidos são publicados artigos em língua inglesa, as palavras-chave pesquisadas também seguiram essa orientação. Desta forma, os termos pesquisados foram Responsabilidade Social Organizacional (Corporate Social Responsibility), Saúde e Segurança Ocupacional (Occupational Health and Safety) e Ergonomia (Ergonomics / Human Factors). 
Visando cumprir o objetivo principal do trabalho descrito inicialmente, descreve-se a aplicação de um modelo Bibliométrico de desenvolvimento considerando a execução das seguintes etapas:

- Definição da amostra da pesquisa;

- Pesquisa na amostra, com as palavras-chave;

- Identificação dos periódicos qualificados segundo a Qualis CAPES;

- Seleção dos artigos indexados à base e publicados nos periódicos anteriormente identificados;

- Classificação dos artigos selecionados em subcategorias;

- Construção de um quadro de análise dos referidos artigos, identificando as interseções entre as subcategorias estudadas.

\section{4- Resultados e discussão}

\section{1- Definição da amostra}

$\mathrm{Na}$ análise dos conceitos de RSO e SSO busca-se identificar as interseções ou incorporações de um conceito pelo outro. A amostra pesquisada corresponde aos documentos indexados nas bases de dados Scopus e Web of Science, com acesso pelo Portal de periódicos Capes em 07 de Junho de 2014.

\section{2- Pesquisa na amostra}

A pesquisa na amostra foi realizada utilizando as seguintes frases: (corporate social responsibility) AND (ergonomics OR human factors OR occupational health and safety). A justificativa pela utilização de tais termos segue a proposta anteriormente ilustrada (Figura 1).

Após a primeira busca, retornaram 242 documentos, sendo 176 da base Scopus e 66 da base Web of Science. A distribuição por tipo de produto está ilustrada na Tabela 1.

Tabela 1 - Distribuição dos registros encontrados por tipo de documento

\begin{tabular}{ccc}
\hline Tipo de documento & Scopus & Web of science \\
\hline Artigos & 114 & 47 \\
\hline Conferências & 24 & 16 \\
\hline Revisões & 17 & 2 \\
\hline Revisões de Conferências & 3 & - \\
\hline Capítulos de livro & 5 & - \\
\hline Notas & 4 & - \\
\hline Resumos & 1 & - \\
\hline Artigos aceitos para publicação & 4 & - \\
\hline Breve levantamento & 2 & 1 \\
\hline Outros & 2 & 66 \\
\hline Total & 176 & \\
\hline
\end{tabular}


(Fonte: $\mathrm{O}$ autor)

Realizou-se então um primeiro refinamento, considerando-se apenas os artigos em periódicos, retornando um total de 161 registros, somando-se as duas bases pesquisadas (114 Scopus e 47 Web of Science). Com o intuito de evitar que artigos comuns às duas buscas fossem computados neste total, realizou-se a filtragem da amostra através do software Endnote X7. Após tal procedimento, identificou-se que 31 artigos eram comuns às duas buscas, delimitando a amostra para 130 artigos. Além disso, o uso do software foi de suma importância para categorizar os artigos segundo ano de publicação e autoria.

\section{3- Identificação dos Periódicos qualificados segundo a Qualis CAPES}

Foram encontrados 107 periódicos indexados às bases Scopus e Web of Science, que atendem aos parâmetros da busca. Optou-se neste artigo em relacionar os periódicos estratificados segundo sua qualidade pela Qualis CAPES na área de Engenharias III (Engenharia de Produção e Sistemas) e Interdisciplinar. A classificação de periódicos é realizada pelas áreas de avaliação e passa por processo anual de atualização. Esses veículos são enquadrados em estratos indicativos da qualidade - A1, o mais elevado; A2; B1; B2; B3; B4; B5; C.

A Tabela 2 nos mostra que, do total de 107 periódicos indexados às bases e que publicaram artigos referentes ao tema pesquisado, 31 eram qualificados pela Qualis CAPES nas áreas anteriormente mencionadas. Observa-se também que do total de 130 artigos coletados na amostra, 49 foram publicados nos referidos periódicos.

Tabela 2 - Periódicos indexados às bases e qualificados segundo a Qualis CAPES na área de Engenharias III e Interdisciplinar

\begin{tabular}{lccc}
\hline \multicolumn{1}{c}{ Periódico } & $\begin{array}{c}\text { Número de } \\
\text { artigos }\end{array}$ & Qualificação & Área \\
\hline Alcohol and Alcoholism & 1 & A1 & Interdisciplinar \\
\hline American Journal of Public Health & 2 & A1 & Interdisciplinar \\
\hline British Food Journal & 2 & B1 & Engenharias III \\
\hline Construction Management and Economics & 1 & B3 & Engenharias III \\
\hline DYNA & 1 & B1 & Engenharias III \\
\hline Ergonomics & 2 & A2 & Engenharias III \\
\hline Global Environmental Change Human and Policy & 1 & A1 & Engenharias III \\
Dimensions & & A1 & Interdisciplinar \\
\hline Globalization and Health & 1 & B1 & Interdisciplinar \\
\hline Health Policy & 1 & B1 & Engenharias III \\
\hline Information Technology and People & 1 & B1 & Engenharias III \\
\hline International Journal of Human Resource & 2 & B1 & Engenharias III \\
\hline Management & 1 & A2 & Engenharias III \\
\hline International Journal of Industrial Ergonomics & & B1 & \\
\hline
\end{tabular}




\begin{tabular}{|c|c|c|c|}
\hline $\begin{array}{l}\text { International Journal of Knowledge Culture and } \\
\text { Change Management }\end{array}$ & 2 & B4 & Engenharias III \\
\hline $\begin{array}{l}\text { International Journal of Quality and Reliability } \\
\text { Management }\end{array}$ & 1 & B2 & Engenharias III \\
\hline Journal of Business Ethics & 9 & B1 & Engenharias III \\
\hline Journal of Cleaner Production & 2 & $\begin{array}{l}\mathrm{A} 2 \\
\mathrm{~A} 1\end{array}$ & $\begin{array}{l}\text { Engenharias III } \\
\text { Interdisciplinar }\end{array}$ \\
\hline Journal of Environmental Management & 2 & $\begin{array}{l}\text { A1 } \\
\text { A1 }\end{array}$ & $\begin{array}{l}\text { Engenharias III } \\
\text { Interdisciplinar } \\
\end{array}$ \\
\hline Lancet & 1 & A1 & Interdisciplinar \\
\hline Latin American Perspectives & 1 & B1 & Engenharias III \\
\hline $\begin{array}{l}\text { New Solutions: A Journal of Environmental and } \\
\text { Occupational Healt Policy }\end{array}$ & 1 & B3 & Engenharias III \\
\hline Perspectives in Biology and Medicine & 1 & B1 & Interdisciplinar \\
\hline PLoS Medicine & 1 & A2 & Interdisciplinar \\
\hline Psychological Reports & 1 & B1 & Interdisciplinar \\
\hline Research in Development Disabilities & 1 & A1 & Interdisciplinar \\
\hline Safety Science & 4 & $\mathrm{~A} 2$ & Engenharias III \\
\hline Scandinavian Journal of Psychology & 1 & B1 & Interdisciplinar \\
\hline Supply Chain Management & 1 & $\mathrm{~A} 2$ & Engenharias III \\
\hline Sustainability & 1 & B5 & Interdisciplinar \\
\hline Tourism Management & 1 & B1 & Interdisciplinar \\
\hline Waste Management & 1 & $\begin{array}{l}\text { A1 } \\
\text { A2 }\end{array}$ & $\begin{array}{l}\text { Interdisciplinar } \\
\text { Engenharias III }\end{array}$ \\
\hline $\begin{array}{l}\text { World Academy of Science, Engineering and } \\
\text { Technology }\end{array}$ & 1 & B3 & Engenharias III \\
\hline
\end{tabular}

(Fonte: $\mathrm{O}$ autor)

Deve-se então, a partir desta análise, maior nível de atenção aos periódicos listados na Tabela 2. Para efeito de maior credibilidade, delimitamos o estudo aos artigos publicados nos referidos periódicos.

\section{4- Seleção dos artigos indexados às bases e publicados nos periódicos qualificados segundo a Qualis CAPES}

Após análise dos 49 artigos anteriormente identificados, foi constatado que 6 artigos não apresentavam congruência com o tema pesquisado. Desta forma, delimitou-se a pesquisa em 43 artigos.

\section{5- Classificação dos artigos selecionados em subcategorias.}

Com o intuito de esmiuçar os temas relativos aos artigos anteriormente selecionados, optou-se por dividi-los em subcategorias, conforme descritas abaixo:

- RSO Externa - dimensão da RSO que agrega um conjunto de práticas que abarcam questões relativas a stakeholders externos, designadamente comunidades locais, clientes, parceiros comerciais, fornecedores, entre outros (VICENTE, 2011); 
- RSO Interna - dimensão da RSO que engloba todas as práticas e políticas adotadas ao nível intra-organizacional (VICENTE, 2011), como o gerenciamento do ambiente e as condições de trabalho, jornada e organização do trabalho, materiais e equipamentos, saúde e segurança do empregado (AL-BDOUR et al, 2010);

- RSO Interna / Externa - artigos que abordam aspectos das duas dimensões da RSO;

- Gestão - artigos que citam a RSO como ferramenta de gestão estratégica da empresa.

O Gráfico 1 sintetiza essa divisão em subcategorias, considerando que dos 43 artigos analisados, 13 abordavam a RSO na sua dimensão externa, 19 na dimensão interna e 11 abordavam aspectos relevantes das duas dimensões.

Gráfico 1 - Distribuição do tema abordado em subcategorias de abordagem, considerando-se os artigos analisados

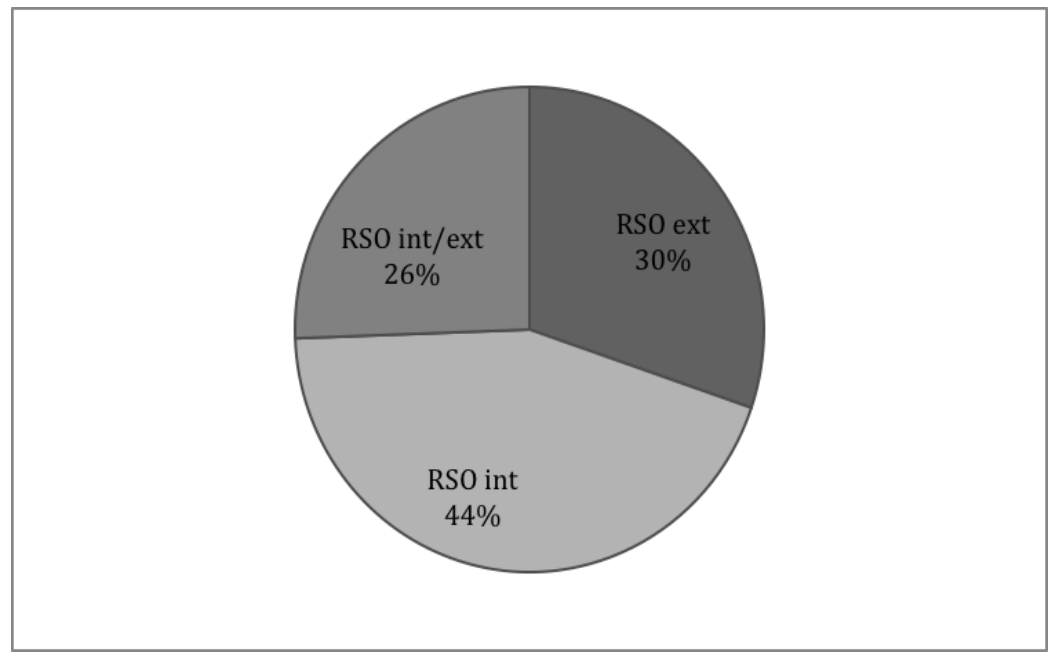

(Fonte: $\mathrm{O}$ autor)

O resultado da análise identificou ainda que, apesar da tentativa de delimitar o escopo da pesquisa com as palavras-chave anteriormente citadas, outros aspectos referentes à RSO podem ser encontrados nos artigos analisados. Além da Ergonomia / SSO, foram identificados também abordagens referentes à Direitos Humanos, Recursos Humanos, Clima Organizacional, Consumidores, Sustentabilidade e Meio Ambiente, Comunidade, Cadeia de Suprimentos e ainda, Gestão Estratégica.

Como o enfoque deste trabalho está na relação entre RSO e SSO, optou-se por dar atenção especial à RSO Interna. Sendo assim, dos 19 artigos que tratam de tal abordagem, 10 referem-se a Ergonomia e SSO, 3 referem-se à Recursos Humanos (RH), 3 discorrem sobre Clima Organizacional, 2 tratam de assuntos referentes à Direitos Humanos e 1 refere-se à RSO como ferramenta de Gestão Estratégica . 
Interna, considerando-se os artigos analisados.

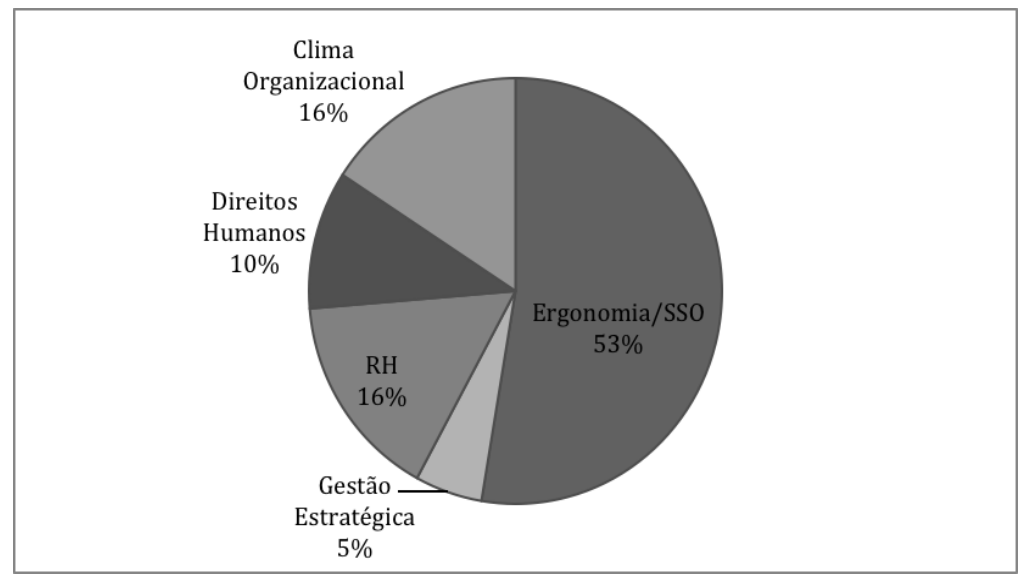

(Fonte: O Autor)

\section{6- Quadro de análise conceitual e de correlação das subcategorias.}

As análises dos artigos selecionados evidencia que existem interseções na abordagem não só das dimensões da RSO, mas também entre os vários aspectos a elas relacionadas. O resultado final deste estudo encontra-se resumido na Tabela 3.

Tabela 3 - Resumo da análise de conteúdo da revisão bibliométrica

\begin{tabular}{|c|c|c|c|}
\hline & \multicolumn{3}{|c|}{ Dimensões da Responsabilidade Social Organizacional } \\
\hline \multirow[t]{2}{*}{$\begin{array}{c}\text { Principais } \\
\text { abordagens } \\
\text { referentes à } \mathrm{RSO}\end{array}$} & RSO Interna & RSO Externa & RSO Interna/Externa \\
\hline & \multicolumn{3}{|c|}{ Autores } \\
\hline Ergonomia / SSO & $\begin{array}{c}\text { Blewett e O'Keeffe (2011), } \\
\text { Brown (2009), Genaidy et al, } \\
\text { (2010), Hadjimanolis e Boustras } \\
\text { (2013), Hart (2010), Koskela } \\
\text { (2014), Metzner e Fisher (2010), } \\
\text { Montero et al (2009), Van } \\
\text { Tulder } \text { et al (2009), Zink (2005) }\end{array}$ & & $\begin{array}{l}\text { Forsman-Hugg et al } \\
\text { (2013), Jones et al } \\
\text { (2005), Petrovic- } \\
\text { Lazarevic (2008) }\end{array}$ \\
\hline $\begin{array}{l}\text { Sustentabilidade / } \\
\text { Meio Ambiente }\end{array}$ & & $\begin{array}{l}\text { Collins (2011), Givel } \\
\text { (2007) }\end{array}$ & $\begin{array}{c}\text { Lozano e Huisingh } \\
\text { (2011), Arruda et al } \\
\text { (2013) }\end{array}$ \\
\hline Dir. Humanos & Jones (2002), Yu (2008) & $\begin{array}{l}\text { Mena et al (2010), } \\
\text { Schrempf (2011) }\end{array}$ & Burke (2010) \\
\hline Clima Organizacional & $\begin{array}{l}\text { Al-Bdour (2010), Chou (2014), } \\
\text { Svensen } \text { et al (2007) }\end{array}$ & & \\
\hline RH & $\begin{array}{l}\text { Gond et al (2011), Parkes e } \\
\text { Davis (2013), Shen (2011) }\end{array}$ & & $\begin{array}{c}\text { Baram (1984), Burke } \\
\text { (2010), Maksimainen e } \\
\text { Saariluoma (2010) }\end{array}$ \\
\hline Consumidores & & $\begin{array}{l}\text { Auger et al (2007), } \\
\text { Baumberg (2009), Chen } \\
\text { et al (2012), Labonté et } \\
\text { al (2011), Watts e } \\
\text { Wyner (2011) }\end{array}$ & \\
\hline $\begin{array}{c}\text { Cadeia de } \\
\text { Suprimentos }\end{array}$ & & Lee e Kim (2009) & \\
\hline
\end{tabular}




\begin{tabular}{c|c|c|c}
\hline Comunidade & & $\begin{array}{c}\text { Garvin } \text { et al (2009), } \\
\text { Givel (2007) }\end{array}$ & Burke (2010) \\
\hline Gestão Estratégica & Metcalf e Benn (2012) & $\begin{array}{c}\text { Ansari e Teimouri } \\
(2009), \text { Fooks } \text { et al } \\
(2011)\end{array}$ & $\begin{array}{c}\text { Asif } \text { et al (2009), Vintró } \\
\text { e Comajuncosa (2010), } \\
\text { Waage (2007) }\end{array}$ \\
\hline
\end{tabular}

(Fonte: O Autor)

\section{5- Conclusões e Sugestões de Novas Pesquisas}

Esta pesquisa propôs como objetivo geral: "Identificar correlações entre os conceitos de RSO e SSO”.

Delinearam-se, também os objetivos específicos:

- Delimitar adequadamente o escopo da coleta de dados, visando coletar uma quantidade significativa de artigos no âmbito da RSO e SSO;

- Categorizar os referidos artigos em subcategorias correlatas com o tema principal de pesquisa;

- Identificar as interseções entre as subcategorias estudadas, com atenção especial à RSO Interna e SSO.

Para isso utilizou-se de um método de pesquisa, baseado na Bibliometria, para auxiliar na construção de conhecimento para a pesquisa científica. Assim, por meio desse método de pesquisa, foi possível selecionar artigos, os autores, os periódicos e as palavras chave mais relevantes para o tema de pesquisa e que formaram um portfólio bibliográfico.

Foram coletados e analisados 49 artigos publicados entre os anos de 1981 e 2014. Destes, 6 foram excluídos da seleção por não apresentarem congruência com o tema pesquisado. Após a revisão de literatura, análise de conteúdo e discussão, apresentados no capitulo 2 e 4 , podem ser apresentadas as seguintes conclusões:

- De acordo com a literatura pesquisada, percebe-se que o conceito de RSO vem consolidando-se como interdisciplinar, multidimensional e associado a abordagem sistêmica, dando relevância às relações com os stakeholders internos e externos (JONES et al, 2005; AL-BDOUR et al, 2010; FORSMAN-HUGG et al, 2013; KOSKELA, 2014) .

- Como consequência, o conceito requer a incorporação à estratégia da organização, com desdobramentos nos eixos econômicos, ambiental e social da operação das organizações (ANSARI; TEIMOURI, 2009; ASIF et al, 2009; METCALF; BENN, 2012).

- Nesse sentido, a abstração ainda presente no conceito de RSO deve dar lugar à construção de indicadores associados às variáveis relacionadas às dimensões e relações da organização com seus diversos stakeholders, principalmente no caso deste estudo, os internos.

- A temática da RSO em sua dimensão interna foi a mais representativa no presente estudo (44\%), certamente devido ao arranjo de palavras-chave utilizado para realizar a pesquisa na 
amostra. Deste percentual, grande parte dos trabalhos abordavam os aspectos da SSO (53\%), fato também justificado pelo referido arranjo. Apesar do maior volume de produção científica com esta temática, nota-se que o surgimento de outras abordagens ainda referentes à RSO Interna, como Clima Organizacional (16\%), Direitos Humanos (10\%), Recursos Humanos (16\%) e Gestão Estratégica (5\%) torna necessário um maior aprofundamento nestas áreas. Para tanto, sugere-se a elaboração de futuras pesquisas com um novo arranjo de palavras-chave.

- O artigo estudou a correlação entre RSO Interna e SSO, que ficou bastante clara nesta pesquisa. Em estudo prévio realizado por Al-Bdour et al (2010), analisando o impacto de cinco aspectos de RSO Interna (incluindo a SSO) em empresas do sector bancário na Jordânia, concluiu-se que todas estas práticas apresentaram correlação significamente positiva com o comprometimento organizacional. Segundo os autores, quando os trabalhadores percebem e sentem que o ambiente de trabalho é adequado, seguro e saudável, os mesmos se sentem valorizados e podem trabalhar mais satisfeitos. Para Hadjimanolis e Boustras (2013), as empresas deveriam estabelecer políticas claras de segurança, objetivando criar um clima positivo e uma cultura de prevenção de riscos, enfatizando o compromisso da gestão com a SSO. Segundo os autores, tais políticas são parte essencial da RSO Interna e fazem sentido para o negócio, pois representam um impacto positivo sobre o desempenho de segurança e na diminuição de custos com acidentes, além de proporcionar a melhoria das atitudes e o aumento da motivação dos trabalhadores.

- Progressos relacionados à política de SSO nas práticas de RSO Interna deveriam incluir, ainda, melhorias em questões de "proteção da vida" e de procedimentos (tais como controle de incêndios e evacuação), eliminação dos riscos de segurança visíveis, melhoras no serviço de limpeza e iluminação, maior oferta de equipamentos de proteção individual e o estabelecimento de programas de SSO e sistemas de gestão (BROWN, 2009). Enfatizando o compromisso da gestão com a SSO, Zink (2005) afirma que a gestão da saúde organizacional requer uma abordagem integrada, com um sistema de metas multidimensional, com a participação dos stakeholders internos e externos e com a compreensão de que a SSO é um meio para garantir a competitividade. Para tanto, o autor cita alguns pontos que podem ser considerados fatores críticos de sucesso na gestão da SSO:
a) A saúde tem que ser parte da política e da estratégia da empresa;
b) Deve haver um forte apoio da alta gerência;
c) É necessária a harmonização com os comitês de segurança industrial existentes;
d) Abordagens organizacionais existentes necessitam de melhoria contínua; 
e) A existência de uma ampla base de dimensões e indicadores de saúde como os sistemas de controle são uma condição prévia relevante;

f) A gestão da saúde na organização tem de ser integrada aos sistemas de gestão existentes ou, pelo menos, estar coordenado com eles;

g) Abordagens de longo prazo devem superar programas de curto prazo.

- A importância da SSO nas práticas de RSO Interna também está evidente no estudo realizado por Koskela (2014). O autor analisou a presença de 35 sub-áreas da SSO nos relatórios de RSO de três empresas de diferentes setores econômicos (aviação, energia e financeiro). Com base nessa análise, a importância da SSO é claramente visível, o que é compreensível em empresas que apresentam riscos físicos eminentes no local de trabalho. Tradicionalmente, o foco da SSO limitava-se na prevenção de acidentes. Entretanto, recentemente a legislação trabalhista reconheceu também a importância dos aspectos psicológicos neste âmbito. O estudo revelou ainda a existência de vários projetos e processos que visam melhorias em SSO, o que pode realmente ser entendido como uma evidência da importância desta área nas empresas estudadas.

- Já no estudo realizado por Forsmann-Hugg et al (2013), a SSO não recebeu a mesma importância. Ao identificar e definir o conteúdo da RSO no contexto da cadeia de abastecimento alimentar, os autores apontaram com base em um processo de pesquisa junto aos stakeholders, sete aspectos da RSO: meio ambiente, segurança do produto, nutrição, bem-estar no trabalho, bem-estar do animal, responsabilidade econômica e bem-estar da comunidade. Neste contexto, o bem-estar ocupacional foi operacionalizado em termos das condições de trabalho, como SSO, salários, igualdade e emprego. Mesmo constituindo uma das principais dimensões da RSO, os autores constataram neste estudo que o bem-estar ocupacional dos trabalhadores na agricultura e no setor alimentar têm recebido menor atenção.

- Em outro estudo, feito com empresas de construção civil na Austrália, Petrovic-Lararevic (2008) analisa a SSO tanto interna quanto externamente. Empregadores, trabalhadores, representantes da comunidade, clientes e fornecedores têm percepções semelhantes acerca do que seja um ambiente de trabalho saudável e seguro, apontando a importância da boa comunicação e de processos de feedback, além da implementação de medidas e treinamento em saúde e segurança. O autor cita neste estudo resultados favoráveis decorrentes da implementação de medidas na SSO internamente, referentes à redução de custos financeiros por afastamento associados com acidentes de trabalho. No entanto, externamente, $75 \%$ das empresas entrevistadas afirmaram que os pequenos sub-empreiteiros não possuem políticas próprias neste sentido. 
- Apesar de o desenvolvimento e a manutenção de um ambiente de trabalho saudável e seguro ser uma obrigação legal, é também uma obrigação social proporcionar tais condições (BLEWEET; O’KEEFFE, 2011). A gestão socialmente responsável mostra seu compromisso, respondendo às necessidades sociais e indo além do mero cumprimento da legislação de segurança por meio de ações positivas para proteger e manter o bem-estar dos trabalhadores (MONTERO et al 2009; HADJIMANOLIS; BOUSTRAS, 2013). No entanto, segundo os autores, diferentes percepções de risco por parte da gestão e dos empregados podem dificultar a implementação de uma política de SSO. Além disso, outros fatores apresentam-se como limitantes para a implantação de tais políticas. Segundo Brown (2009), os modelos tradicionais de SSO envolvem uma ação centrada em três atores principais - o governo, os empregadores e os trabalhadores. Entretanto, um destes pilares (os trabalhadores) estão esquecidos por completo, e os outros dois são divergentes. Hadjimanolis e Boustras (2013) defendem que a gestão da SSO deveria contar com a participação dos trabalhadores, através da formação, partilha de informação e participação nas decisões relacionadas com a segurança.

- É atribuída ao empregador a responsabilidade de proporcionar um ambiente de trabalho seguro e saudável ao trabalhador (HART, 2009). Um estudo realizado na Austrália por Bleewet e O'Keeffe (2011), por exemplo, mostra que todas as 9 jurisdições australianas são semelhantes na medida em que obrigam os empregadores a gerenciar os riscos trabalhistas de maneira sistêmica. A lei exige que o empregador prepare e mantenha atualizada uma declaração escrita com as disposições, práticas e procedimentos no local de trabalho, protegendo a saúde e segurança dos empregados.

- A SSO consiste em um dos temas mais importantes da Ergonomia, neste contexto relacionada com a resolução de problemas técnicos, ou seja, a ênfase tem sido a de evitar os trabalhadores fiquem feridos ao manusear máquinas e equipamentos no trabalho (ZINK, 2005). O autor cita que, que surge como proposta a ser utilizada para maximização do desempenho das tecnologias de processo e de produto (METCALF; BENN, 2012; GENAIDY et al, 2010), além de proporcionar ao trabalhador melhores condições de trabalho. Todavia, os trabalhos analisados não mencionaram especificamente qual(is) o(s) tipo(s) de intervenção ergonômica utilizado(s).

A pesquisa realizada se justifica pela contribuição prática e teórica ao tema de Gestão de SSO no contexto da RSO Interna. A contribuição teórica se explicita pelo fornecimento de um perfil dos artigos científicos que tratam sobre o tema e a prática pela disponibilização de um método de 
pesquisa que possibilita identificar, filtrar e quantificar por meio de critérios pré-estabelecidos, os artigos necessários e suficientes para a elaboração do constructo teórico de pesquisa.

A análise da produção científica sobre RSO possibilitou algumas reflexões que demonstram a necessidade de novas pesquisas para esse campo de estudo, principalmente por dois motivos distintos: (a) o caráter neófito da área no Brasil, que no momento encontra-se em expansão e necessita de maiores estudos, e (b) o crescimento no interesse sobre a temática, com foco na sua dimensão interna.

Vale ressaltar que tais lacunas se configuram como oportunidades de pesquisas e publicações futuras, bem como, possibilidades de contribuição para o campo. Como sugestão para pesquisas futuras, torna-se importante ressaltar os seguintes tópicos: (a) ampliação da base de análise a partir da coleta de artigos em anais de outros periódicos e congressos; (b) replicar o presente estudo em anos posteriores, buscando analisar a evolução do tema; (c) empreender estudos em RSO Interna e SSO que possam contribuir para a evolução do conhecimento em âmbito internacional, levando em consideração o mainstream da área de gestão da produção.

Como qualquer estudo acadêmico, este também possui limitações. A pesquisa contou com a utilização de duas bases de busca: a Scopus e a Web os Science. Embora sejam duas das maiores bases mundiais de artigos, neste escopo não foi analisado a produção nacional, pois as bases citadas indexam somente periódicos internacionais. Vale também ressaltar que a mesma pesquisa pode ser realizada com diferentes combinações de palavras-chave, fato este que certamente contribuirá para o enriquecimento do banco de artigos. Além disso, a quantidade de periódicos analisados também pode ser considerado como uma limitação ao estudo.

Numa perspectiva de escopo, tais decisões foram tomadas levando em consideração os recursos disponíveis, fato que foge do poder de intervenção dos pesquisadores. Contudo, tais limitações podem ser resolvidas em trabalhos futuros, conforme já sugerido.

Por se tratar de uma pesquisa científica teórica, este artigo oferece uma abordagem generalista sobre o tema. Sendo assim, sua contribuição fundamental consiste no auxilio ao entendimento do ambiente científico em que este assunto se insere, permitindo o embasamento de estudos futuros nesta área.

\section{Abstract}

Socially responsible organizations should prize, at first, for the health and welfare of its workforce (employees), even before performing actions that benefit the general public. In this sense, the study of Ergonomics can be of fundamental importance in the construct of favorable conditions for work and improvements in health and worker safety. The intersection between Organizational Social Responsibility (OSR) and Occupational Health and Safety (OHS) proposes an important topic to be addressed in current and future research, to the extent that aspects relating to working conditions are increasingly latent. This research can be characterized as a desk research, in other words, was based on secondary data and aimed to identify correlations between the concepts of SSO and RSO. In 
total 49 articles published between 1981 and 2014 in the journals of the Capes Portal, and bases Scopus and Web of Science were analyzed. It was observed that the main results of the internal dimension RSO represents $44 \%$ of published articles and $53 \%$ of these focus on Ergonomics and OHS. Some research gaps appeared after this study, a fact that suggests future research on the subject.

Key-words: internal organizational social responsibility; management of occupational health and safety; sustainability.

\section{Referências}

AL-BDOUR, A.A.; NASRUDDIN, E.; LIN, S.K. The relationship between internal corporate social responsibility and organizational commitment within the banking sector in Jordan. World Academy of Science, Engineering and Technology, v.4, n.7, p.262-281, 2010

ANSARI, M. E.; TEIMOURI, H. The role of corporate social responsibility strategy on success of Multi-National organizations. International Journal of Knowledge, Culture and Change Management, v 9, n.1, p. 63-74, 2009

ARRUDA, L. R.; LAMEIRA, V.J.; QUELHAS, O.L.G.; PEREIRA, F.N. Sustainability in the Brazilian Heavy Construction Industry: An Analysis of Organizational Practices. Sustainability, v.5, n.10, p. 4312-4328, 2013 crossref

ASIF, M.; DE BRUJIN, E.J.; FISSCHER, O.A.M.; SEARCY, C.; STEENHUIS, H.J. Process embedded design of integrated management systems. International Journal of Quality and Reliability Management, v.26, n.3, p. 261282,2009

crossref

ASSOCIAÇÃO BRASILEIRA DE NORMAS TÉCNICAS (ABNT). NBR ISO 26000:2010. Diretrizes sobre responsabilidade social. Rio de Janeiro: ABNT, 2010

AUGER, P.; DEVINNEY, T.M.; LOUVIERE, J.J. Using best-worst scaling methodology to investigate consumer ethical beliefs across countries. Journal of Business Ethics, v.70, n.3, p. 299-326, 2007 crossref

BAUMBERG, B. How will alcohol sales in the UK be affected if drinkers follow government guidelines? Alcohol and Alcoholism, v.44, n.5, p. 523-528, 2009 cross ref

BLEWETT, V.; O'KEEFFE, V. Weighing the pig never made it heavier: Auditing OHS, social auditing as verification of process in Australia. Safety Science, v.49, n.7, p. 1014-1021, 2011 crossref

BROWN, G. Genuine worker participation-an indispensable key to effective global OHS. New solutions: a journal of environmental and occupational health policy, v.19, n.3, p. 315-333, 2009

BURGESS, K.; SINGH, P. J.; KOROGLU, R. Supply chain management: a structured literature review and implications for future research. International Journal of operations and Production Management, v. 26, n. 7, p. 703-729, 2006 crossref

BURKE, B. J. Cooperatives for "fair globalization"? Indigenous people, cooperatives, and corporate social responsibility in the Brazilian Amazon. Latin American Perspectives, v.37, n.6, p. 30-52, 2010 cross ref

CHEN, C. C.; LIN, S.Y.; CHENG, C.H.; TSAI, C.C. Service quality and corporate social responsibility, influence on post-purchase intentions of sheltered employment institutions. Research in Developmental Disabilities, v.33, n.6, p. $1832-1840,2012$ crossref

CHOU, C. J. Hotels' environmental policies and employee personal environmental beliefs: Interactions and outcomes. Tourism Management, v.40, p. 436-446, 2014 crossref

COLLINS, M. B. Risk-based targeting: Identifying disproportionalities in the sources and effects of industrial pollution. American Journal of Public Health, v.101, (suppl. 1), p. 231-237, 2011 crossref

COSTA, H. ISO 26000 - Norma Internacional de Responsabilidade Social: um guia para entendê-la melhor. São Paulo: Instituto Observatório Social, 2011 
CRUZ, F.J.A.; CABRAL, A.C.A.; PESSOA, M.N.M; SANTOS, S.M. Relações entre responsabilidade social interna e comprometimento organizacional: um estudo em empresas prestadoras de serviços. Revista Brasileira de Administração Científica, Aquidabã, v.3, n.3, p.163-182, 2012

DUL, J., NEUMANN, W.P. Ergonomics contributions to company strategies. Applied Ergonomics, v.40, p. 745-752, 2009 cross ref

FARIA, A.; SAUERBRONN, F. F. A responsabilidade social é uma questão de estratégia? Uma abordagem crítica. Rev. Adm. Pública [online]. v.42, n.1, 2008

FOOKS, G. J.; GILMORE, A.B.; SMITH, K.E.; HOLDEN, C.; LEE, K. Corporate social responsibility and access to policy élites: An analysis of tobacco industry documents. PLoS Medicine, v.8, n.8, p. 1-12, 2011 crossref

FORSMAN-HUGG, S.; KATAJAJUURI, J.M.; RIIPI, I.; JÄRVELÄ, K.; TIMONEN, P. Key CSR dimensions for the food chain. British Food Journal, v.115, n.1, p. 30-46, 2013 crossref

FUKUNAGA, E.M. Responsabilidade social interna: uma contribuição ao debate. Revista de Ciências Gerenciais. v. 13, n. 18, p.195-206, 2009

GARVIN, T., McGEE, T.K.; SMOYER-TOMIC, K.E.; AUBYNN, E.A. Community-company relations in gold mining in Ghana. Journal of Environmental Management, v.90, n.1, p. 571-586, 2009 cross ref

GENAIDY, A. M., RINDER, M.M.; SEQUEIRA, R.; A-REHIM, A. The role of human-at-work systems in business sustainability: perspectives based on expert and qualified production workers in a manufacturing enterprise. Ergonomics, v.53, n.4, p. 559-585, 2010 crossref

GIVEL, M. Motivation of chemical industry social responsibility through Responsible Care. Health Policy, v.81, n.1, p. 85-92, 2007 cross ref

GOND, J.P.; IGALENS, J.; SWAEN, V.; AKREMI, A.E. The human resources contribution to responsible leadership: an exploration of the CSR-HR interfaces. Journal of Business Ethics, v.98, (suppl. 1), p. 115-132, 2011 cross ref

HADJIMANOLIS, A.; BOUSTRAS, G. Health and safety policies and work attitudes in Cypriot companies. Safety Science, v.52, p.50-56, 2013 crossref

HART, S. M. Self-regulation, Corporate Social Responsibility, and the Business Case: Do they Work in Achieving Workplace Equality and Safety? Journal of Business Ethics, v.92, n.4, p. 585-600, 2010 crossref

JONES, N. J. Sex and racial differences in preference for a caring morality in a corporate environment that promotes diversity and equal opportunity. Psychological Reports, v.91, n.2, p. 641-649, 2002 crossref

JONES, P; COMFORT, D; HILLIER, D; EASTWOOD, I. Corporate Social Responsibility: a case study of the UK's leading food retailers. British Food Journal, v.107, n.6, p. 423-435, 2005 cross ref

KOGI, K. Pratical ways to facilitate ergonomics improvements in occupational health practice. Human Factors: The Journal of the Human Factors and Ergonomics Society, v. 54, n. 6, p. 890-900, 2012 crossref

KOSKELA, M. Occupational health and safety in corporate social responsibility reports. Safety Science, n.68, p. 294308,2014 cross ref

LABONTÉ, R.; MOHINDRA, K.S.; LENCUCHA, R. Framing international trade and chronic disease. Globalization and Health, v.7, n.21, p. 1-15, 2011 crossref

LEE, K. H.; KIM, J.W. Current status of CSR in the realm of supply management: The case of the Korean electronics industry. Supply Chain Management, v.14, n.2, p. 138-148, 2009crossref

LOZANO, R.; HUISINGH, D. Inter-linking issues and dimensions in sustainability reporting. Journal of Cleaner Production, v.19, n.2-3, p. 99-107, 2011 cross ref

MAKSIMAINEN, J.; SAARILUOMA, P. How human resource management and human capital management influence corporate social responsibility (CSR). International Journal of Knowledge, Culture and Change Management, v.10, n.5, p. 111-125, 2010 
McCAUGHEY, D.; DELLIFRAINE, J.L.; McGHAN, G.; BRUNING, N.S. The negative effects of workplace injury and illness on workplace safety climate perceptions and health care worker outcomes. Safety Science, v. 51, n.1, p.138147,2013 crossref

MENA, S., DE LEEDE, M.; BAUMANN, D.; LINDEMAN, S.; McSHANE, L. Advancing the Business and Human Rights Agenda: Dialogue, Empowerment, and Constructive Engagement. Journal of Business Ethics, v.93, n.1, p. 161188,2010 crossref

METCALF, L.; BENN, S. The Corporation is Ailing Social Technology: Creating a 'Fit for Purpose' Design for Sustainability. Journal of Business Ethics, v.111, n.2, p. 195-210, 2012 crossref

METZNER, R.J.; FISHER, F.M. Fatigue and workability in Brazilian textile companies in diferente corporate social responsibility score groups. International Journal of Industrial Ergonomics, v. 40, n.3, p.289-294, 2010cross ref

MONTERO, M.J.; ARAQUE, R.A.; REY, J.M. Occupational health and safety in the framework of corporate social responsibility. Safety Science, v. 47, n.10, p.1440-1445, 2009crossref

MUELLER, K., HATTRUP, K., SPIESS, S.O., LIN-HI, N. The effects of corporate social responsibility on employees' affective commitment: a cross-cultural investigation. Journal of Applied Psychology, v. 97, n.6, p.1186-1200, 2012 crossref

PARKES, C.; DAVIS, A.J. Ethics and social responsibility - do HR professionals have the 'courage to challenge' or are they set to be permanent 'bystanders? International Journal of Human Resource Management, v.24, n.12, p. 24112434, 2013crossref

PETROVIC-LAZAREVIC, S. The development of corporate social responsibility in the Australian construction industry. Construction Management and Economics, v.26, n.2, p. 93-101, 2008crossref

RICART, S.L.S.I., VIDAL, M.C.R., BONFATTI, R.J. Evaluation and control of ergonomics actions in federal public servisse: the case of FIOCRUZ-RJ. Work, v.41, n. suppl 1, p. 532-538, 2012

SCHREMPF, J. Nokia Siemens Networks: Just Doing Business - or Supporting an Oppressive Regime? Journal of Business Ethics, v.103, n.1, p. 95-110, 2011crossref

SHEN, J. Developing the concept of socially responsible international human resource management. International Journal of Human Resource Management, v.22, n.6, p. 1351-1363, 2011crossref

SRIVASTAVA, S. K. Green supply-chain management: a state-of-the-art literature review. International Journal of Management Reviews, v. 9, n. 1, p. 53-80, 2007crossref

SVENSEN, E.; NESET, G.; ERIKSEN, H.R. Factors associated with a positive attitude towards change among employees during the early phase of a downsizing process. Scandinavian Journal of Psychology, v.48, n.2, p.153-159, 2007 cross ref

VAN TULDER, R.; VAN WIJK, J; KOLK, A. From Chain Liability to Chain Responsibility. Journal of Business Ethics, v.85, p. 399-412, 2009 cross ref

VICENTE, A.L.F.M.S., REBELO, T.M.M.S.D, AGOSTINHO, C.F. Relação das práticas de responsabilidade social interna nas organizações com a satisfação no trabalho e as intenções de saída: O papel mediador do ajustamento pessoaorganização. Psychologica, v.55, p. 369-384, 2011 cross ref

VILELA, R.A.G., ALMEIDA, I.M., MENDES, R.W.B. Da vigilância para prevenção de acidentes de trabalho: contribuição da ergonomia da atividade. Ciência \& Saúde Coletiva, v.17, n.10, p. 2817-2830, 2012 cross ref

VINTRÓ, C.; COMAJUNCOSA, J. Corporate social responsibility in the mining industry: Criteria and indicators. DYNA (Colombia), v.77, n.161, p. 31-41, 2010

WAAGE, S. A. Re-considering product design: a practical "road-map" for integration of sustainability issues. Journal of Cleaner Production, v.15, n.7, p. 638-649, 2007 crossref

WATTS, S.; WYNER, G. Designing and theorizing the adoption of mobile technology-mediated ethical consumption tools. Information Technology \& People, v.24, n.3, p. 257-280, 2011crossref 
YU, X. M. Impacts of corporate code of conduct on labor standards: A case study of Reebok's athletic footwear supplier factory in China. Journal of Business Ethics, v.81, n.3, p. 513-529, 2008

ZINK, K. J. From industrial safety to corporate health management. Ergonomics, v.48, n.5, p. 534-546, 2005 crossref

\section{Dados dos autores}

\section{Nome Completo: Sávio Luís Oliveira da Silva}

Filiação Institucional:UFF Universidade Federal Fluminense

Departamento:Engenharia Civil

Função Ou Cargo Ocupado: Mestrando em Sistemas De Gestão

Endereço Completo Para Correspondência: Rua Prof. Fernando José De Almeida 200 Casa 1 -

Piratininga, Niterói , RJ - CEP: 24358-085

Telefones Para Contato: (21) 98153-1946 / (21) 2622-6825

e-mail: contato@savioluis.com

\section{Nome Completo: Osvaldo Luiz Gonçalves Quelhas}

Filiação Institucional: UFF Universidade Federal Fluminense

Departamento: Engenharia Civil

Função Ou Cargo Ocupado: Professor Titular

Endereço Completo Para Correspondência: Rua Geógrafo Amora, 501, Rua 2 Lote 4 Quadra I,

Condominio Residencial Camboatá, Piratininga, Niteroi, Rj. Cep: 24.350590

Telefones Para Contato:21 999973493 Ou 2127098421

e-mail:quelhas@latec.uff.br

Submetido em: 08-04-2015

Aceito em: 07-05-2015 